This item was submitted to Loughborough's Research Repository by the author.

Items in Figshare are protected by copyright, with all rights reserved, unless otherwise indicated.

\title{
A comparison of methods used for inducing mental fatigue in performance research: individualised, dual-task and short duration cognitive tests are most effective
}

\section{PLEASE CITE THE PUBLISHED VERSION}

https://doi.org/10.1080/00140139.2019.1687940

\section{PUBLISHER}

Informa UK Limited, trading as Taylor \& Francis Group

\section{VERSION}

AM (Accepted Manuscript)

\section{PUBLISHER STATEMENT}

This is an Accepted Manuscript of an article published by Taylor \& Francis in Ergonomics on 18 November 2019, available online: http://www.tandfonline.com/10.1080/00140139.2019.1687940.

\section{LICENCE}

CC BY-NC-ND 4.0

\section{REPOSITORY RECORD}

O'Keeffe, Kate, Simon Hodder, and Alexander Lloyd. 2019. "A Comparison of Methods Used for Inducing Mental Fatigue in Performance Research: Individualised, Dual-task and Short Duration Cognitive Tests Are Most Effective". figshare. https://hdl.handle.net/2134/11357849.v1. 


\section{A comparison of methods used for inducing mental fatigue in performance research: Individualised, dual-task and short duration cognitive tests are most effective.}

Kate O'Keeffe a, Simon Hodder ${ }^{\text {a }}$ and Alex Lloyd ${ }^{\text {a* }}$

${ }^{a}$ Environmental Ergonomics Research Centre, Loughborough University, UK.

* Corresponding Author:

Alex Lloyd, PhD.

Environmental Ergonomics Research Centre

James France Building, Design School

Loughborough University

Leicestershire, LE11 3TU

Email: A.Lloyd@lboro.ac.uk

Dr. Simon Hodder: $\underline{\text { S.Hodder@,1boro.ac.uk }}$

Kate O'Keeffe: k.e.okeeffe@,lboro.ac.uk 


\title{
A comparison of methods used for inducing mental fatigue in performance research: Individualised, dual-task and short duration cognitive tests are most effective.
}

\begin{abstract}
Despite research indicating the negative impact that mental fatigue has on physical and cognitive performance, whether this is a result of mental fatigue or a state of under-arousal remains unclear. The current research aimed to explore the effectiveness of the methods being used to induce mental fatigue. Twelve participants attended six sessions in which two cognitive tests, the AX-continuous performance test (AX-CPT) and the TloadDback test, were compared for their effectiveness in inducing mental fatigue. Both tests were set at a standard processing speed $(1.2 \mathrm{~ms})$ for two conditions, and a further condition involved the individualisation of the TloadDback test. Participants presented significantly higher physiological and psychological arousal $(p<0.05)$ in the individualised dual task test compared to the AX-CPT. The individualised TloadDback test is a more effective method of inducing mental fatigue compared to the AX-CPT, as it sustains physiological arousal whilst inducing measurable reductions in mental resources.
\end{abstract}

Keywords: mental fatigue, arousal, galvanic skin conductance, heart rate variability, cognitive loading

Practitioner Summary: Mental fatigue negatively impacts physical and cognitive performance. It is unclear whether the current methods being used to induce mental fatigue are effective. This study compared different methods and confirmed that short, individualised and dual task tests are most effective for inducing mental fatigue whilst maintaining arousal.

Word count: 5638 


\section{Introduction}

Mental fatigue impacts performance in many professions (Boksem \& Tops 2008; Gergelyfi et al. 2015) and is one of the most common causes of accidents and errors in the modern world (Lew and Qu 2014; Tanaka 2015) Research conducted on the characteristics of mental fatigue define it as a psychobiological state (Marcora et al. 2009) that is characterized subjectively by a lack of energy and lethargy, and/or objectively as a reduction in the ability to complete cognitive tasks (Boksem \& Tops 2008; Qian et al. 2015). Mental fatigue manifests as a result of utilising and overusing the brains resources. This leads to a reduced competence of the brain to undertake cognitive workloads efficiently (Tanaka 2015). Due to the accumulative decrease in productivity and increase in errors, both physical (Marcora et al. 2009; Smith et al. 2016) and cognitive performance can be reduced as a result of mental fatigue (Holtzer et al. 2011; Tanaka et al. 2014). It is therefore necessary to explore methods to counteract the negative impact of mental fatigue on human performance.

All definitions in the current literature surrounding the impact of mental fatigue on physical and cognitive performance outline the importance of cognitive activity over prolonged periods of time for effective inducement of mental fatigue (van der Linden et al. 2003; Boksem \& Tops 2008; Marcora et al. 2009). However, the level and duration of cognitive activity required to induce mental fatigue is unclear. In their review, Van Cutsem et al. (2017) implicitly emphasized the importance of task duration for inducing subjective mental fatigue i.e. cognitive tests that were shorter than 30 -minutes were excluded, and of the eleven articles that were included, seven articles used cognitive tests that lasted for a period of 90-minutes or longer. The main test utilised in these studies was the 90-minutes version of the AX-continuous performance test (AX-CPT), which requires 
low levels of cognitive activity and processing. In contrast to this Boksem \& Tops (2008) present that even short tasks can lead to measurable evidence of reduced cognitive resources. Furthermore, as discussed by Borragán et al. (2017), mental fatigue is independent from general fatigue and sleepiness. When taken together, these studies question whether current methods that involve prolonged effort are inducing mental fatigue per se, or rather a psychological and physiological state of under-arousal (i.e. sleepiness, boredom).

A key psychophysiological state that the present study aims to explore in correlation with sleepiness and performance levels, is arousal. In performance research, arousal is used as both a psychological and physiological state of alertness, the feeling of being activated or energized, and it is not synonymous with stress or anxiety (Perkins and Wilson 2001). To achieve consistent optimal performance, research has indicated the need to achieve optimal arousal states prior to performance (Ruiz et al. 2017). Previous research investigating mental fatigue, has utilised a 'pre-performance' style methodology, whereby prior to physical performance participants are required to undertake a cognitive test with the goal to induce a state of mental fatigue (Marcora et al. 2009; Pageaux et al. 2014; Martin et al. 2016; Smith et al. 2016). Similarly, for control conditions, studies have often utilised time matched, emotionally neutral documentaries. However, previous research does not determine whether participants felt 'emotionally neutral' towards the documentaries used. Therefore, it may be that existing methodologies used prior to endurance and maximal exertion tests cause participants to experience states of underarousal. Hence, mental fatigue may not be the sole stressor impacting the subsequent physical performance. 
Individual differences in cognitive abilities are also well documented. Fundamental differences across individuals encompass the traits of knowledge, skills, aptitude and achievement (Sackett et al. 2017). Cognitive control therefore is not a unitary ability and it is erroneous to judge an individual's cognitive ability based on standardised tests (Gonthier et al. 2016). Many previous studies inducing mental fatigue have not taken individual factors into account. Therefore, reliable accounts of whether similar levels of cognitive effort and thereby mental fatigue were facilitated for all participants remains unclear (Borragán et al. 2017).

This investigation took a neuropsychological perspective based on the theory of cognitive loading in conjunction with the time-based resource-sharing model (Barrouillet et al. 2004) to work towards exploring an alternative method of inducing mental fatigue effectively in psychophysiology and performance research. Cognitive load theory suggests that the working memory is limited (Paas and Ayres 2014) and the time-based resource-sharing model (TBRS) (Borragán, et al. 2017) implies that to induce mental fatigue, what matters is the amount of time a person has to process the incoming stimulus. This model presents the idea that performance is related to the amount of time needed for the attention to process ongoing information (Barrouillet et al. 2004). To address the limitations of tests like the AX-CPT, the present study employed the 'TloadDback' which incorporates both the cognitive loading and time-based resource-sharing paradigms (Borragán et al. 2017).

To explore the effectiveness of the current methods used for inducing mental fatigue, this investigation compared the commonly used AX-CPT and the TloadDback tests for inducing mental fatigue, as well as two methods of control comparisons, a standard set- 
up protocol, and a time-matched documentary. Three hypotheses were constructed: 1. Mental fatigue will be induced using both tests, however the TloadDback test will maintain arousal; 2. The individualised TloadDback test will indicate the greatest levels of mental fatigue inducement, and higher levels of both subjective and objective arousal; 3. The time-matched documentary control condition will present lower levels of subjective and objective arousal when compared to a standard set-up protocol control. An understanding into the psychophysiological outcomes of these hypotheses will provide important information into the study of the effect of mental fatigue on physical performance and provide insight into the effectiveness of the current methods being used for inducing mental fatigue in performance research.

\section{Methods}

\subsection{Procedure}

Participants were recruited from Loughborough University. Twelve participants aged $26.5 \pm 3.12$ years, including seven females and five males, were required to be physically active and not have any history of neurological injury. The study procedures were approved by the Loughborough University ethics committee, and all procedures were conducted in compliance with the Declaration of Helsinki (1964). All participants were paid for their participation pro rata. All trials were conducted at the Loughborough University Environmental Ergonomics Research Centre. The study consisted of six trials, including one familiarisation session and five main experimental trials, each conducted on separate days.

\subsection{Familiarisation and individualisation session}

In the familiarisation session, participants were accustomed to both the subjective (see section: Subjective Ratings) and objective measures (see sections: Galvanic Skin 
Conductance and Heart Rate Variability) of the study. Following this, familiarisation and individualisation of the TloadDback cognitive test then took place. There were four stages in the individualisation process. As the TloadDback test is a dual task test, the two individual tasks were practiced separately first, and then together.

The first stage involved practicing the primary task which was a parity judgement test, where participants distinguished between odd and even numbers - when an even number was presented, the participant pressed the number 2, and when an odd number was presented, participants pressed the number 3 . This task was performed using the right hand. The secondary task involves the classic n-back paradigm - a series of letters were presented, and participants responded by pressing the space bar, with the left hand, when the letter currently being presented was identical to the letter that was presented immediately before. Numbers and letters were alternated in sequence. The third stage was a combined practice, with both the parity test and n-back test. Participants were only allowed to proceed to the next round if they successfully achieved $>85 \%$ performance. Prior to starting the final stage, the individualisation process (Figure 1A), participants took a long break to ensure that the onset of mental fatigue was limited. Glasses of cold water were provided to maintain stimulation between stages.

The individualisation stage was conducted in blocks, with each block consisting of 30 letters and 30 numbers. The initial stimulus speed was 1400-msecs. To progressively increase the cognitive demand of the test, the stimulus speed was reduced by 100 -msecs per block. Each participant took a break of at least one minute between blocks. Participants could only start the next block when a state of mental alertness was restored (based on subjective measures of mental fatigue, which were taken at the end of each 
break). The individual stimulus time detection (i.e. shortest stimulus rate a person can process the incoming information at) was found when a person was no longer able to maintain accuracy greater than $85 \%$, signifying that a participant had reached their cognitive load limit (Borragán et al. 2017). This speed was then used for the individualised TloadDback test in the main trials. Borragán et al. (2017) induced a low cognitive load by using a stimulus time detection that was $50 \%$ longer than the speed attained when participants reached their cognitive load limit (i.e. high cognitive load). However, in the current study, the TloadDback standardised condition was performed with a standardised letter/number presentation time of $1200-\mathrm{ms}$ in order to effectively compare to the $\mathrm{AX}-\mathrm{CPT}$ which has been incorporated in previous research using this speed (Marcora et al. 2009; Brownsberger et al. 2013).

(Insert Figure 1.)

\subsection{Main experimental trials}

Of the experimental conditions, two were designed to recreate potential 'control' conditions for mental fatigue studies (Figure 1B), and the remaining three experimental conditions aimed to induce mental fatigue using different cognitive tests (Figure 1C). Thus, the five experimental interventions were:

(1) Set-Up Control, in which participants sat resting for two minutes only. This condition was selected to recreate the process of preparation for an experimental study only;

(2) Documentary control, in which participants watched two documentaries in succession, lasting a total of 90-minutes as per previous research (Marcora et al. 2009; 
Brownsberger et al. 2013). The documentaries were: The History of the Ferrari: The Definitive story (Boulevard Entertainment 2006) and The Venice Simplon Orient Express (Beardsall and Garofalo 2004).

(3) AX-CPT, a 90-minute single-task continuous performance test. Participants were required to press the space bar when the letter $\mathrm{X}$ followed the letter $\mathrm{A}$, among a series of randomly generated letter sequences. This test had a standardised letter presentation time of 1200-ms across all participants as per previous research (Marcora et al. 2009; Brownsberger et al. 2013).

(4) TloadDback STD, a 16-minute dual task test, where the participant must perform two cognitive tasks at one time as described above (see: Familiarisation and Individualisation Session). In the current study the TloadDback STD was performed with a standardised letter/number presentation time of $1200-\mathrm{ms}$ in order to effectively compare to the AXCPT, and elicit a low cognitive load (Borragán et al. 2017).

(5) TloadDback INDV, the same 16-minute dual task test as noted above, with the exception that the letter/number presentation time was individualised based on performance in the familiarisation trial (see section: Familiarisation and Individualisation Session). All participants in this study had individualised speeds faster than the standardised speed $(<1200-\mathrm{ms})$. Therefore, the individualised speeds induced a higher cognitive load on the individual than in the standardised condition (Borragán et al. 2017). 
Pre- and post- each intervention subjective ratings of mental fatigue, mood, sleepiness, and motivation were assessed (see section: Subjective Ratings). Participants underwent the standard 2-minutes baseline protocol to assess measures of GSC and HRV. Both were measured continuously during each test.

Participants were required not to consume any caffeinated drinks or food for up to two hours prior to participation in the study. Trials were counterbalanced, and participants were requested to come in at the same time of day for each trial, in a similar physical and psychological state as the previous trial. In the familiarisation session participants filled out the Morningness-Eveningness Questionnaire (MEQ) which is a self-assessment questionnaire used to determine an individuals' peak alertness in relation to their circadian rhythms (Horne and Östberg 1976). Participants are categorised by five different 'types' ranging from 'definitely morning type', 'moderately morning type', 'intermediate (neither) type', 'moderately evening type', and 'definitely evening type'. Participants in this study identified themselves as; 'moderate morning types', 'moderate evening types' and 'intermediate (neither) type'. Trials were scheduled according to each participants' relative peak. In all conditions, participants entered the testing room and sat down at the computer table, after which, all necessary forms were filled out prior to commencing the trial. Tests were conducted on a standard desktop computer (HewlettPackard). The height of the monitor display (Hewlett-Packard, LA2205wg, 22inch, 1680x1050 resolution) was adjusted for each participant so that the stimulus appeared at eye level. All test stimuli (letters and numbers) appeared in the centre of the screen, in white font (Times New Roman) against a black background. 


\subsection{Subjective ratings}

Subjective scales were used to monitor self-reported scores of mental fatigue, mood, sleepiness, and motivation. A 10-point visual analogue scale was used to assess mental fatigue with 0 representing "no fatigue" and 10 representing "worst possible fatigue". In the familiarisation session, participants were briefed on how to differentiate mental fatigue from physiological fatigue and sleepiness. Participants were told to assess their ability to focus or concentrate and the presence or lack of mental fogginess. Smith et al. (2019) recently reported that a visual analogue scale is the most effective method for assessing mental fatigue. Motivation is an essential component of mental fatigue as it is required for a participant to sustain as much attention to the task as possible throughout the course of the trial. Motivation was manipulated through offering a monetary bonus as a result of attaining over $50 \%$ on average across all tests and monitored via the task and success motivation scales (Matthews et al. 2013). Mood change is also characteristic of mental fatigue (Boksem and Tops 2008). Moods were assessed using the Brunel Mood Scale (BRUMS) where fatigue, vigour, confusion, depression, tension and anger were monitored pre- and post-conditions (Terry et al. 2003). Subjective scores of sleepiness using The Stanford Sleepiness Scale (SSS) (Hoddes et al. 1973) was also implicated.

\subsection{Galvanic skin conductance}

GSC was measured by applying two electrodes to the glabrous skin (Boucsein et al. 2012) on the inside of the foot which passed a low voltage through the skin which recorded skin conductance. The foot was chosen as the preferred site as both hands were needed to perform the cognitive test. Prior to the application of the electrodes, the skin was cleaned and sterilized with $70 \%$ ethanol wipes. Before starting each trial, a 2-minute baseline recording was taken to ensure that the electrodes were responding and to determine the 
baseline skin conductance for pre- and post- analysis. To test the electrodes, participants were asked to take two deep breaths at 30 -seconds and 90 -seconds. Taking a fast and deep breath elicits an electrodermal response in the conductance reading (Boucsein et al. 2012). This is in response to activating the sympathetic nervous system which causes a small increase in GSC, hence increasing the electrical conductivity of the skin. To minimize artifacts in the data, participants were instructed prior to measurement to breathe normally, keep movement to a minimum, try not to speak throughout the trial and to sit in as comfortable a position as possible.

GSC was measured in micro-Siemens $(\mu \mathrm{S})$ and was analyzed using the MatLAB compatible software, Ledalab (Benedek and Kaernbach 2010). Arousal was measured by analyzing the phasic level of the galvanic skin conductance data as it related to the activation of the sympathetic nervous system. Raw data was imported from the Biopac output files (ACQ). Once imported, the raw data was down-sampled from $2000 \mathrm{~Hz}$ to $10 \mathrm{~Hz}$ to speed up data processing. Three pre-processing steps were undertaken prior to analysis. Step 1; the data was corrected for artifacts by removing any physical movements that resulted in clear changes in electrodermal activity. These artefacts were recorded by the researcher with their time of occurrence and duration noted throughout the trial by observing the live GSC trace. Examples of movements causing artifacts were; augmented breathing such as yawning, stretches, movement in the chair and tapping of the foot. Step 2; a low pass filter was applied to eliminate any high frequency noise components. Step 3; the data was smoothed using the Gaussian window smoothing technique with a window width of 8 samples, to account for further movement artefacts. The data was then analyzed through continuous decomposition analysis (Benedek and Kaernbach 2010), which 
decomposes the skin conductance data into continuous tonic and phasic activity. This method is based on standard deconvolution which is both fast and robust to artifacts.

\subsection{Heart rate variability}

Heart rate variability was collected using an Equivital (Hidalgo, UK) life monitor system. The system collected an array of variables including heart rate, breathing rate, ECG data, skin temperature and interbeat intervals (IBI). Interbeat interval data represents the duration between beats, R-R intervals. Once collected, the data was then analyzed using Kubios software (Tarvainen et al. 2014). The extracted IBI data was transferred from excel to a text file where it was then imported to the Kubios HRV standard software. Once imported, the data was passed through a very low artefact correction threshold (Tarvainen et al. 2014). In the time domain, to assess vagal tone, the root mean square of the successive difference (RMSSD) was calculated and has been shown to be highly correlated with parasympathetic activity (Laborde et al. 2017). A second variable extracted was the PNN50 which is also associated with parasympathetic activation and is the percentage of adjacent beats $\mathrm{NN}$ intervals that differ from each other by more than 50-ms (Laborde et al. 2017).

\subsection{Data analysis}

A one-way multivariate analysis of variance (MANOVA) was used to determine differences between groups of related dependent variables. One-way, repeated measures analysis of variances (ANOVAs) were used for further analysis of the differences between conditions, with subsequent analysis of pairwise comparisons across conditions

when the MANOVA was significant. Paired sample T-tests were used to determine differences from pre-to post-test within conditions. A 95\% confidence level was used to 
test significance $(p<0.05)$. When Mauchly's Test of Sphericity was significant $(p<0.05)$, the Greenhouse-Geisser adjustment was used. Data are presented as mean $\pm \mathrm{SD}$.

\section{Results}

\subsection{Objective mental fatigue: TloadDback performance}

The AX-CPT presented significantly higher performance results, with an error rate of $0.1 \% \pm 0.01$ when compared to both the standardised and individualised TloadDback ( $\mathrm{p}$ $<0.001)(7 \% \pm 0.04$ and $25 \% \pm 0.1$ respectively $)$. The maintenance of test performance below $85 \%$ indicates participants sustained a higher cognitive load throughout the test. (Figure 2A).

(Insert Figure 2.)

\subsection{Objective arousal}

There were no significant differences in baseline measures of objective arousal across conditions: GSC, $p=0.17$; RMSSD, $p=0.90$; PNN50, $p=0.80$. A main effect of condition was seen on GSC ( $\mathrm{p}=0.006)$, where the individualised TloadDback displayed a significant increase in skin conductance when compared to both the standardised TloadDback $(+97 \%, \mathrm{p}=0.007)$ and the AX-CPT $(+165 \%, \mathrm{p}=0.015)($ Figure $2 \mathrm{~B})$. Overall mean heart rate was higher in the individualised TloadDback, $(M=78.5 \pm 9.8 \mathrm{bpm})$, when compared to the standardised TloadDback $(\mathrm{M}=72.2 \pm 9.3 \mathrm{bpm})$, the AX-CPT $(72.4 \pm 10.8 \mathrm{bpm})$ and the documentary control $(\mathrm{M}=72.4 \pm 8.8 \mathrm{bpm}), \mathrm{p} \leq 0.032$. A significant decrease was seen in the RMSSD between the standardised TloadDback ( 54.8 $\pm 28.9)$, the AX-CPT $(55.1 \pm 19.2)$ and the individualised TloadDback $(37.7 \pm 26.3), \mathrm{p} \leq$ 0.013 (Figure 2C). PNN50 scores displayed a similar trend with higher scores in the 
standardised TloadDback $(25.3 \pm 19.2)$ and the set-up control $(23.6 \pm 17.6)$ when compared to the individualised TloadDback (16.7 \pm 18.5$), \mathrm{p} \leq 0.02$ (Figure 2D).

\subsection{Subjective mental fatigue: Visual analogue scale}

There were no significant differences in subjective pre-test mental fatigue scores across conditions, $\mathrm{p}=0.652$. Subjective post-test mental fatigue scores indicated significant differences pre vs post in all conditions, $p<0.001$, except the set-up control $(0.08 \pm 0.2$, $\mathrm{p}=0.339$ ). Across conditions there were also significant differences between post-test mental fatigue scores $(\mathrm{p}<0.001)$. The individualised TloadDback produced the highest subjective scores of mental fatigue $(4.4 \pm 2.7)$ (Figure $3 \mathrm{~A})$.

(Insert Figure 3.)

\subsection{Stanford sleepiness scale}

There were no significant differences in pre-test sleepiness scores across conditions, $\mathrm{p}=$ 0.726. Post-test sleepiness scores produced significant differences in all conditions when compared to pre-test sleepiness scores $(\mathrm{p}<0.001)$ except in the set-up control, $(1.5 \pm 0.5$, $\mathrm{p}=0.151)$ (Figure 3B). Across conditions, post-test scores saw further significant differences $(p<0.001)$. The AX-CPT reported the highest sleepiness scores $(4.4 \pm 1.4)$ and was significantly higher than the individualised TloadDback $(2.7 \pm 0.9, \mathrm{p}=0.007)$.

\subsection{Subjective motivation}

Motivation questionnaires were analyzed in terms of task motivation and success motivation (Matthews et al., 2013). Results indicated that participants significantly decreased in task motivation in all conditions $(p \leq 0.025)$, except the set-up control $(p=$ 
0.062) (Figure 3C). The AX-CPT presented the greatest decrease in task motivation with a $41 \%$ reduction from pre-test task motivation $(20.2 \pm 3.4)$ to post-test task motivation $(12 \pm 7), \mathrm{p}<0.001$. Additionally, the AX-CPT induced a significant decrease in task motivation in comparison to all other conditions, $\mathrm{p} \leq 0.03$. Conversely, the individualised TloadDback presented only a $10 \%$ decrease in task motivation from pre-test $(21.7 \pm 3.4)$ to post-test $(19.5 \pm 4.3)$.

With regards success motivation, the individualised TloadDback, the standardised TloadDback and the set-up control presented no significant changes in success motivation from pre to post-test, $\mathrm{p} \geq 0.125$ (Figure 3D). Both the AX-CPT and the documentary control presented significant decreases in success motivation from pre to post-test, $\mathrm{p} \leq$ 0.008. The documentary control presented a 55\% decrease in success motivation when pre-test $(16.3 \pm 6)$ was compared to post-test $(7.4 \pm 7.3)$ and compared to the individualised TloadDback, the standardised TloadDback and the set-up control presented a significant reduction in success motivation, post-test, $p \leq 0.04$. Likewise, the AX-CPT presented a reduction in success motivation by $30 \%$ and was significantly reduced when compared to the individualised TloadDback test, $\mathrm{p}=0.05$.

\subsection{Subjective mood: Brunel mood scale}

There were no significant differences in baseline ratings of fatigue $(p=0.53)$, vigour $(p$ $=0.64)$, confusion $(p=0.08)$, depression $(p=0.62)$, tension $(p=0.46)$ or anger $(p=0.53)$. Across all conditions, fatigue was significantly increased from pre-test to post-test, $\mathrm{p} \leq$ 0.012 , except in the set-up control, $\mathrm{p}=0.33$ (Figure 3E). Likewise, vigour significantly decreased in all conditions, $p \leq 0.009$, except in the set-up control, $p=0.249$ (Figure 3F). The largest increase in fatigue was seen in the AX-CPT with a $190 \%$ increase from pre- 
test $(5.2 \pm 7.4)$, to post test $(15.1 \pm 15.2)$. Similarly, the AX-CPT reported a $70 \%$ decrease in vigour scores from pre-test $(49.5 \pm 14.7)$ to post-test $(15.1 \pm 14.1)$. Furthermore, the AX-CPT presented significantly lower post-test vigour when compared to all other conditions, $\mathrm{p}<0.037$.

The TloadDback tests were significantly lower in post-test fatigue scores when compared to the AX-CPT, $\mathrm{p} \leq 0.001$. Other significant mood differences post-test included confusion, which increased from pre-test $(1.5 \pm 2.8)$ to post-test $(12.5 \pm 13.8), \mathrm{p}=0.031$, in the individualised TloadDback. In the AX-CPT, depression increased from pre-test $(1.04 \pm 9.9)$ to post-test $(9.9 \pm 10.8), \mathrm{p}=0.009$, and anger from pre-test $(1.5 \pm 3.8)$ to post-test $(7.7 \pm 7.9), \mathrm{p}=0.032$.

\subsection{Overview of results}

To summarize, a ranked referencing system presents the main findings from the analysis (Table 1). The three methods for inducing mental fatigue and the documentary control were ranked in order from 1 being the 'worst' to 4 being the 'best' for inducing mental fatigue and maintaining arousal across all measures. Reverse ranking is in place for the measures of VASF, SSS, fatigue (BRUMS), RMSSD and PNN50, as the lower the reported scores, the better the performance of the condition for inducing mental fatigue whilst maintaining arousal. The set-up control was excluded as it was the 'best' ranked or not applicable across all measures.

(Insert Table 1.) 


\section{Discussion}

\subsection{Current methods and mental fatigue}

This study aimed to explore the effectiveness of the current methods used for inducing mental fatigue. Through investigating the differences in both objective and subjective arousal measures, the results concluded that the single task AX-CPT left participants in a state of under-arousal post-test. Conversely, both the standardised and individualised version of the dual task TloadDback test left participants in a neutral state of arousal posttest. Subjective levels of mental fatigue post-test confirm that prolonged attention to a cognitive task will induce mental fatigue. However, subjective sleepiness scores confirm a much higher level of sleepiness in the AX-CPT when compared to the TloadDback tests. This indicates that mental fatigue, independent of sleepiness, was not achieved by the AX-CPT, but was achieved to a much greater extent by the TloadDback tests. The results confirm the first hypothesis of this study, that both tests induced a subjective perception of mental fatigue, yet the TloadDback tests maintained higher levels of arousal.

\subsection{Performance and arousal}

Overall, the individualised version of the TloadDback test produced the highest levels of mental fatigue and maintained greater levels of arousal. When compared to the standardised TloadDback test, the individualised version sustained higher levels of arousal, mental fatigue and motivation levels. Performance in the tests presented minimal error rates in both the AX-CPT (1\%) and the standardised TloadDback test $(7 \%)$ characteristic of a low cognitive load (Borragán et al. 2017). The individualised TloadDback sustained performance below $85 \%$, indicative of participants engaging in a higher cognitive load for the duration of the test. The maintenance of a higher cognitive 
load has previously been shown to induce mental fatigue faster and to a greater extent (Borragán et al. 2017).

Objective levels of arousal were also indicated by galvanic skin conductance and heart rate variability. The individualised TloadDback condition elicited the greatest skin conductance implying greater arousal (Benedek \& Kaernbach, 2010) than all other conditions except the set-up control. Similarly, heart rate variability, reported as RMSSD and PNN50, presented higher levels of arousal. These higher levels of arousal are indicative of cognitive stress due to a maladaptive response in combatting the stress placed on the executive function. This is reflected in lower levels of RMSSD and PNN50 in the individualised TloadDback test. The AX-CPT presented higher levels of both RMSSD and PNN50 which suggests an adaptive response due to the lack of stimulation that the AX-CPT presented to participants. These results confirm the second hypothesis that the individualised TloadDback test would indicate greater levels of mental fatigue, and both subjective and objective arousal. It is therefore proposed that increased attention should be applied in methodological designs when using cognitive tasks to induce mental fatigue, such that all participants are classified as individual respondents to the mental fatigue protocol being incorporated, i.e. tests are individualised.

\subsection{Mood and motivation}

Motivation is an important variable in facilitating the maintenance of attention and focus to complete a cognitive test. In the review by Van Cutsem et al. (2017), the importance of considering paradigms such as motivation and boredom is highlighted when tasks that incur long durations are utilised. Results from this study concluded that over the course of the AX-CPT, both task and success motivation scores decreased notably. On the 
contrary, whilst the standardised version of the TloadDback test did decrease minimally over time in both task and success motivation, the individualised version of the TloadDback test increased over time in success motivation and decreased minimally in task motivation. This indicates that participants were considerably more motivated in the individualised TloadDback test on comparison to the other conditions, and hence limiting the decrease in vigour and attention over time that long duration and non-stimulating tasks induce.

All mental fatigue trials induced substantial mood change. A large decrease in vigour is seen in the AX-CPT, whilst in comparison, the individualised TloadDback test saw only a minimal decrease. Regarding general fatigue, the AX-CPT produced considerably higher levels compared to any of the other tests, with a $471 \%$ increase from pre- to post test scores, confirming states of under-arousal post-test. Furthermore, the AX-CPT saw an increase in depression and anger, whilst the individualised TloadDback test saw a notable increase in confusion post-test. Hence, the mood changes characteristic of the AX-CPT further clarifies the lack of psychological and physiological stimulation the test induces, therefore impeding arousal.

\subsection{Control conditions and arousal}

It was hypothesised that the time-matched documentary control condition would elicit lower levels of arousal when compared to the set-up control. Results from this study indicate that the documentary control elicited a similar arousal response to the AX-CPT. When compared to the standard set-up control, the documentary control presented higher levels of mental fatigue, sleepiness, decreases in motivation, increases in general fatigue and a substantial decrease in vigour. Galvanic skin conductance analysis concluded much 
lower arousal levels in the documentary control. With regards heart rate variability both the set-up control and documentary control presented high levels of RMSSD and PNN50 indicating lower levels of cognitive stress or parasympathetic activation. The high levels of RMSSD and PNN50 in the set-up control were unexpected but can be attributed to the required deep breathing protocol during the 2-mins baseline procedure. Deep and slowpaced breathing has been shown to increase heart rate variability (Russo, Santarelli, and O’Rourke 2017).

Thus, the documentary control appeared to leave participants in a state of under arousal, which like the AX-CPT could impact detrimentally on performance. In addition, previous research does not consider whether participants felt 'emotionally neutral' towards the documentary. In this study, a questionnaire for explorative purposes was included to assess whether participants would remain emotionally neutral from such documentary topics. In conclusion, $60 \%$ of participants reported that they would be bored from watching a documentary on trains, and $25 \%$ reported that they would be bored from watching a documentary on Ferraris. This therefore indicated that most participants would not remain in a state of neutral emotion hence consideration into what 'emotionally neutral' signifies is necessary.

\subsection{Implications}

Previous studies investigating the impact of mental fatigue on physical performance have largely found that it does negatively impact performance (Marcora et al. 2009; Pageaux et al. 2014; Smith et al. 2016; Van Cutsem et al. 2017; McMorris et al. 2018). However, the implications of this study question whether mental fatigue was induced independently of sleepiness, and if the mental fatigue test itself was the main impactor on physical 
performance. This study confirms that the AX-CPT may induce mental fatigue, but it is not independent of sleepiness. This non-individualised test induces psychological states of boredom, demotivation, depressive and anger mood states, loss of attention and underarousal, confirmed both subjectively and objectively. Implications of this study suggest a better method for inducing mental fatigue to reduce the impact of under-arousal on physical performance and isolating the effect of mental fatigue. Furthermore, this study confirms that it is not a necessity to utilise tests that are equal to or longer than 30 -minutes. What is necessary, is that researchers looking to explore mental fatigue per se, consider methodologies from the cognitive sciences. A better understanding in both domains will yield more synchronized and valuable research in the psychophysiology domain. Previous research has incorporated the use of brain monitoring strategies such as electroencephalography (EEG) (Trejo et al. 2007; Wascher et al. 2014; Trejo et al. 2015; Van Cutsem et al., 2018) and functional near-infrared spectroscopy (fNIRS) (Tseng et al. 2018; Borragán et al. 2019) to quantify mental fatigue. Hence, future research should utilise such methods to objectively inform researchers whether the brain regions associated with mental fatigue are activated. Not only would this compliment subjective measures of mental fatigue, but it would also ensure that it has been induced effectively prior to assessing its impact on behavioural or physical outcomes.

Declaration of Interest: The authors declare they have no conflicts of interests and this research did not receive any specific grant from funding agencies in the public, commercial, or not-for-profit sectors. 


\section{References}

Barrouillet, Pierre, Sophie Bernardin, and Valérie Camos. 2004. "Time Constraints and Resource Sharing in Adults' Working Memory Spans." Journal of Experimental Psychology: General. American Psychological Association. doi:10.1037/00963445.133.1.83.

Beardsall, lyn, and Robert Garofalo. 2004. The Venice Simplon Orient Express. Pegasus Entertainment.

Benedek, Mathias, and Christian Kaernbach. 2010. “A Continuous Measure of Phasic Electrodermal Activity." Journal of Neuroscience Methods 190 (1). Elsevier B.V.: 80-91. doi:10.1016/j.jneumeth.2010.04.028.

Boksem, Maarten A S, and Mattie Tops. 2008. "Mental Fatigue: Costs and Benefits." Brain Research Reviews 59 (1). Elsevier B.V.: 125-139. doi:10.1016/j.brainresrev.2008.07.001.

Borragán, Guillermo, Carlos Guerrero-Mosquera, Céline Guillaume, Hichem Slama, and Philippe Peigneux. 2019. "Decreased Prefrontal Connectivity Parallels Cognitive Fatigue-Related Performance Decline after Sleep Deprivation. An Optical Imaging Study.” Biological Psychology 144 (March). Elsevier: 115-124. doi:10.1016/j.biopsycho.2019.03.004.

Borragán, Guillermo, Hichem Slama, Mario Bartolomei, and Philippe Peigneux. 2017. “Cognitive Fatigue: A Time-Based Resource-Sharing Account.” Cortex 89 (October). Elsevier Ltd: 71-84. doi:10.1016/j.cortex.2017.01.023.

Boucsein, Wolfram, Don C. Fowles, Sverre Grimnes, Gershon Ben-Shakhar, Walton T. Roth, Michael E. Dawson, and Diane L. Filion. 2012. "Publication Recommendations for Electrodermal Measurements." Psychophysiology 49 (8): 1017-1034. doi:10.1111/j.1469-8986.2012.01384.x.

Brownsberger, J, A Edwards, R Crowther, and D Cottrell. 2013. "Impact of Mental Fatigue on Self-Paced Exercise Brownsberger 2013.” International Journal of Sports Medicine 34 (12): 1029-1036. 
Entertainment, Boulevard. 2006. The History of the Ferrari: The Definitive Story. Boulevard Entertainment Ltd.

Gergelyfi, Mónika, Benvenuto Jacob, Etienne Olivier, and Alexandre Zénon. 2015. "Dissociation between Mental Fatigue and Motivational State during Prolonged Mental Activity." Frontiers in Behavioral Neuroscience 9 (July): 1-15. doi:10.3389/fnbeh.2015.00176.

Gonthier, Corentin, Brooke N. Macnamara, Michael Chow, Andrew R.A. Conway, and Todd S. Braver. 2016. "Inducing Proactive Control Shifts in the AX-CPT." Frontiers in Psychology 7 (NOV): 1-14. doi:10.3389/fpsyg.2016.01822.

Hoddes, E., V. Zarcone, H. Smythe, R. Phillips, and W. C. Dement. 1973. "Quantification of Sleepiness: A New Approach.” Psychophysiology 10 (4): 431436. doi:10.1111/j.1469-8986.1973.tb00801.x.

Holtzer, Roee, Melissa Shuman, Jeannette R. Mahoney, Richard Lipton, and Joe Verghese. 2011. "Cognitive Fatigue Defined in the Context of Attention Networks." Aging, Neuropsychology, and Cognition 18 (1): 108-128. doi:10.1080/13825585.2010.517826.

Horne, J A, and O Östberg. 1976. “A Self-Assessment Questionnaire to Determine Morningness-Eveningness in Human Circadian Rhythms.” International Journal of Chronobiology. Östberg, O.: Department of Human Work Sciences, University of Lulea, Lulea, Sweden, S-95187: Gordon and Breach Science Pub Ltd.

Laborde, Sylvain, Emma Mosley, and Julian F. Thayer. 2017. "Heart Rate Variability and Cardiac Vagal Tone in Psychophysiological Research - Recommendations for Experiment Planning, Data Analysis, and Data Reporting." Frontiers in Psychology 8 (FEB): 1-18. doi:10.3389/fpsyg.2017.00213.

Lew, Fui Ling, and Xingda Qu. 2014. "Effects of Mental Fatigue on Biomechanics of Slips.” Ergonomics 57 (12). Taylor \& Francis: 1927-1932. doi:10.1080/00140139.2014.937771.

Marcora, Samuele M, Walter Staiano, and Victoria Manning. 2009. "Mental Fatigue Impairs Physical Performance in Humans.” Journal of Applied Physiology 
(Bethesda, Md. : 1985) 106 (3): 857-864. doi:10.1152/japplphysiol.91324.2008.

Martin, Kristy, Walter Staiano, Paolo Menasp??, Tom Hennessey, Samuele Marcora, Richard Keegan, Kevin G. Thompson, David Martin, Shona Halson, and Ben Rattray. 2016. "Superior Inhibitory Control and Resistance to Mental Fatigue in Professional Road Cyclists." PLoS ONE 11 (7): 1-15. doi:10.1371/journal.pone.0159907.

Matthews, Gerald, James Szalma, April Rose Panganiban, Catherine Neubauer, and Joel S. Warm. 2013. Profiling Task Stress With the Dundee Stress State Questionnaire. Psychology of Stress. doi:10.1016/j.foodres.2014.12.027.

McMorris, Terry, Martin Barwood, Beverley J. Hale, Matt Dicks, and Jo Corbett. 2018. "Cognitive Fatigue Effects on Physical Performance: A Systematic Review and Meta-Analysis.” Physiology and Behavior 188 (February): 103-107. doi:10.1016/j.physbeh.2018.01.029.

Paas, Fred, and Paul Ayres. 2014. "Cognitive Load Theory: A Broader View on the Role of Memory in Learning and Education.” Educational Psychology Review 26 (2): 191-195. doi:10.1007/s10648-014-9263-5.

Pageaux, Benjamin, Romuald Lepers, Kristina C. Dietz, and Samuele M. Marcora. 2014. "Response Inhibition Impairs Subsequent Self-Paced Endurance Performance.” European Journal of Applied Physiology 114 (5): 1095-1105. doi:10.1007/s00421-014-2838-5.

Perkins, D, and GV Wilson. 2001. "The Effects of Elevated Arousal and Mood on Maximal Strength Performance in Athlete.” Journal of Applied Sport Psychology 13 (September 2000): 239-259.

Qian, Shaowen, Min Li, Guoying Li, Kai Liu, Bo Li, Qingjun Jiang, Li Li, Zhen Yang, and Gang Sun. 2015. "Environmental Heat Stress Enhances Mental Fatigue during Sustained Attention Task Performing: Evidence from an ASL Perfusion Study." Behavioural Brain Research 280: 6-15. doi:10.1016/j.bbr.2014.11.036.

Ruiz, Montse C., John S. Raglin, and Yuri L. Hanin. 2017. "The Individual Zones of Optimal Functioning (IZOF) Model (1978-2014): Historical Overview of Its 
Development and Use." International Journal of Sport and Exercise Psychology 15 (1). Taylor \& Francis: 41-63. doi:10.1080/1612197X.2015.1041545.

Russo, Marc A., Danielle M. Santarelli, and Dean O'Rourke. 2017. “The Physiological Effects of Slow Breathing in the Healthy Human.” Breathe 13 (4): 298-309. doi:10.1183/20734735.009817.

Sackett, P R, F Lievens, and C H Van Iddekinge. 2017. "Individual Differences and Their Measurement: A Review of 100 Years of Research." Journal of Applied Psychology 102 (3): 254-273. doi:10.1037/ap10000151.supp.

Smith, Mitchell R., Rifai Chai, Hung T. Nguyen, Samuele M. Marcora, and Aaron J. Coutts. 2019. "Comparing the Effects of Three Cognitive Tasks on Indicators of Mental Fatigue." The Journal of Psychology 0 (0). Routledge: 1-25. doi:10.1080/00223980.2019.1611530.

Smith, Mitchell R., Aaron J. Coutts, Michele Merlini, Dieter Deprez, Matthieu Lenoir, and Samuele M. Marcora. 2016. "Mental Fatigue Impairs Soccer-Specific Physical and Technical Performance." Medicine and Science in Sports and Exercise 48 (2): 267-276. doi:10.1249/MSS.0000000000000762.

Tanaka, Masaaki. 2015. "Effects of Mental Fatigue on Brain Activity and Cognitive Performance: A Magnetoencephalography Study.” Anatomy \& Physiology s4: 0-4. doi:10.4172/2161-0940.S4-002.

Tanaka, Masaaki, Akira Ishii, and Yasuyoshi Watanabe. 2014. "Neural Effect of Mental Fatigue on Physical Fatigue: A Magnetoencephalography Study." Brain Research 1542. Elsevier: 49-55. doi:10.1016/j.brainres.2013.10.018.

Tarvainen, Mika P., Juha Pekka Niskanen, Jukka A. Lipponen, Perttu O. Ranta-aho, and Pasi A. Karjalainen. 2014. "Kubios HRV - Heart Rate Variability Analysis Software." Computer Methods and Programs in Biomedicine 113 (1). Elsevier Ireland Ltd: 210-220. doi:10.1016/j.cmpb.2013.07.024.

Terry, P. C., A. M. Lane, and G. J. Fogarty. 2003. "Construct Validity of the Profile of Mood States - Adolescents for Use with Adults." Psychology of Sport and Exercise 4 (2): 125-139. doi:10.1016/S1469-0292(01)00035-8. 
Trejo, Leonard J., Karla Kubitz, Roman Rosipal, Rebekah L. Kochavi, and Leslie D. Montgomery. 2015. "EEG-Based Estimation and Classification of Mental Fatigue.” Psychology 6 (5): 572-589. doi:10.4236/psych.2015.65055.

Trejo, Leonard J, Kevin Knuth, Raquel Prado, Roman Rosipal, Karla Kubitz, Rebekah Kochavi, Bryan Matthews, and Yuzheng Zhang. 2007. "EEG-Based Estimation of Mental Fatigue: Convergent Evidence for a Three-State Model.” In Foundations of Augmented Cognition, edited by Dylan D Schmorrow and Leah M Reeves, 201211. Berlin, Heidelberg: Springer Berlin Heidelberg. doi:DOI: 10.1007/978-3-54073216-7_23.

Tseng, Yi-Li, Chia-Feng Lu, Shih-Min Wu, Sotaro Shimada, Ting Huang, and Guan-Yi Lu. 2018. "A Functional Near-Infrared Spectroscopy Study of State Anxiety and Auditory Working Memory Load.” Frontiers in Human Neuroscience 12 (August). doi:10.3389/fnhum.2018.00313.

Van Cutsem, Jeroen, Kevin De Pauw, Luk Buyse, Samuele Marcora, Romain Meeusen, and Bart Roelands. 2017. "Effects of Mental Fatigue on Endurance Performance in the Heat." Medicine and Science in Sports and Exercise 49 (8): 1677-1687. doi:10.1249/MSS.0000000000001263.

Van Cutsem, Jeroen, Kevin De Pauw, Samuele Marcora, Romain Meeusen, and Bart Roelands. 2018. “A Caffeine-Maltodextrin Mouth Rinse Counters Mental Fatigue." Psychopharmacology 235 (4). Psychopharmacology: 947-958. doi:10.1007/s00213-017-4809-0.

van der Linden, Dimitri, Michael Frese, and Theo F. Meijman. 2003. "Mental Fatigue and the Control of Cognitive Processes: Effects on Perseveration and Planning." Acta Psychologica 113 (1): 45-65. doi:10.1016/S0001-6918(02)00150-6.

Wascher, Edmund, Björn Rasch, Jessica Sänger, Sven Hoffmann, Daniel Schneider, Gerhard Rinkenauer, Herbert Heuer, and Ingmar Gutberlet. 2014. "Frontal Theta Activity Reflects Distinct Aspects of Mental Fatigue.” Biological Psychology 96 (1). Elsevier B.V.: 57-65. doi:10.1016/j.biopsycho.2013.11.010. 


\section{Tables}

Table 1. Results table presenting conditions ranked against each other for performance across different measures. Generally, a rank of 1 is considered the 'worst' and a score of 4 is considered the 'best' for inducing mental fatigue and maintaining arousal. In the shaded grey rows, reverse ranking exists whereby a lower reported score of the measure is ranked the 'best' (4) and a higher score is ranked the 'worst' (1). Measures include galvanic skin conductance (GSC), heart rate (HR), root mean square of the differences (RMSSD), the percentage of NN intervals differing by greater than 50-ms (PNN50), mental fatigue (VASF), sleepiness (SSS), task and success motivation, and vigour and fatigue using the Brunel Mood Scale (BRUMS). 


\section{Figures}

Figure 1. Schematics: 1A. Mental fatigue familiarisation and individualisation protocol;

1B. Control conditions; 1C. Mental fatigue conditions. GSC = Galvanic Skin

Conductance, HRV = Heart Rate Variability, SSS = Stanford Sleepiness Scale, BRUMS

$=$ Brunel Mood Scale, VASF $=$ Visual Analogue Scale for Fatigue.

Figure 2. Objective Measures: 2A. Test performance; 2B. Galvanic skin conductance presented in micro-Siemens $(\mu \mathrm{S}) ; 2 \mathrm{C}$. Heart rate variability (HRV) is represented by the root mean square of the successive differences (RMSSD); and 2D. The percentage of adjacent $\mathrm{NN}$ intervals differing by greater than 50-ms (PNN50); $*=$ significant difference between conditions ( significance $=\mathrm{p}<0.05$ ).

Figure 3. Subjective Measures: 3A. Mental fatigue measured using a 0-10 visual analogue scale; 3B. Stanford Sleepiness Scale; 3C. Task motivation; 3D. Success motivation; 3E. Brunel mood scale for fatigue; 3F. Brunel mood scale for vigour; \# = significant difference from set-up control, $\phi=$ significant difference from the documentary control, $\varnothing=$ significant difference from AX-CPT, $\bullet=$ significant difference from the TloadDback INDV; $*$ = significant difference from pre-test to posttest $(\mathrm{p}<0.05)$.

The percentage of adjacent NN intervals differing by greater than 50-ms (PNN50) 
Table 1.

\begin{tabular}{|c|cccc|}
\hline Measure & Documentary & AX-CPT & $\begin{array}{c}\text { TloadDback } \\
\text { STD }\end{array}$ & $\begin{array}{c}\text { TloadDback } \\
\text { INDV }\end{array}$ \\
\hline GSC & 1 & 2 & 3 & 4 \\
HR & 2 & 3 & 1 & 4 \\
RMSSD & 3 & 1 & 2 & 4 \\
PNN50 & 3 & 2 & 1 & 4 \\
Mental Fatigue (VASF) & 1 & 2 & 3 & 4 \\
Sleepiness (SSS) & 2 & 1 & 3 & 4 \\
Task Motivation & 2 & 1 & 3 & 4 \\
Success Motivation & 1 & 2 & 3 & 4 \\
Vigour (BRUMS) & 2 & 1 & 3 & 3 \\
Fatigue (BRUMS) & 2 & 1 & 4 & $\mathbf{3 9}$ \\
\hline Total & $\mathbf{1 9}$ & $\mathbf{1 6}$ & $\mathbf{2 6}$ & \\
\hline
\end{tabular}




\section{Figure 1.}

A

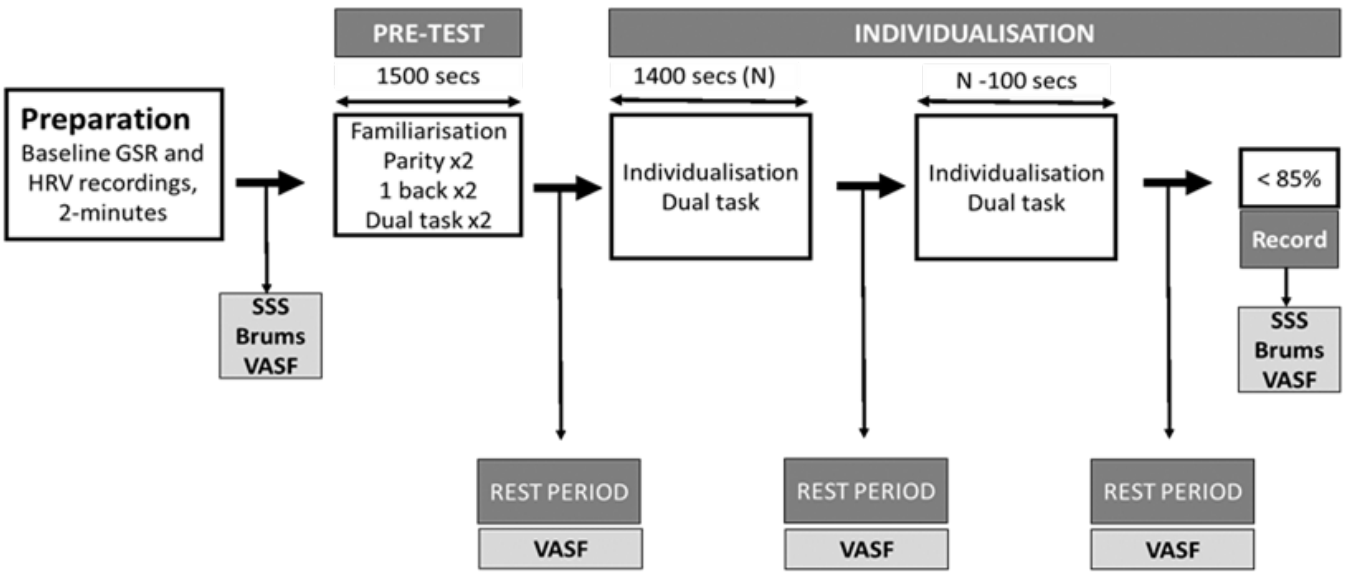

B

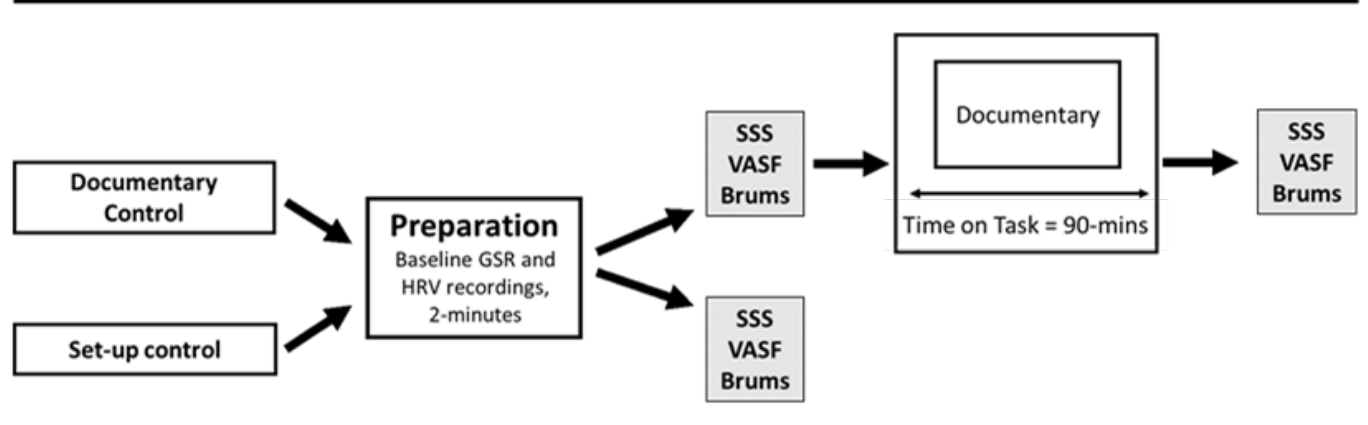

C

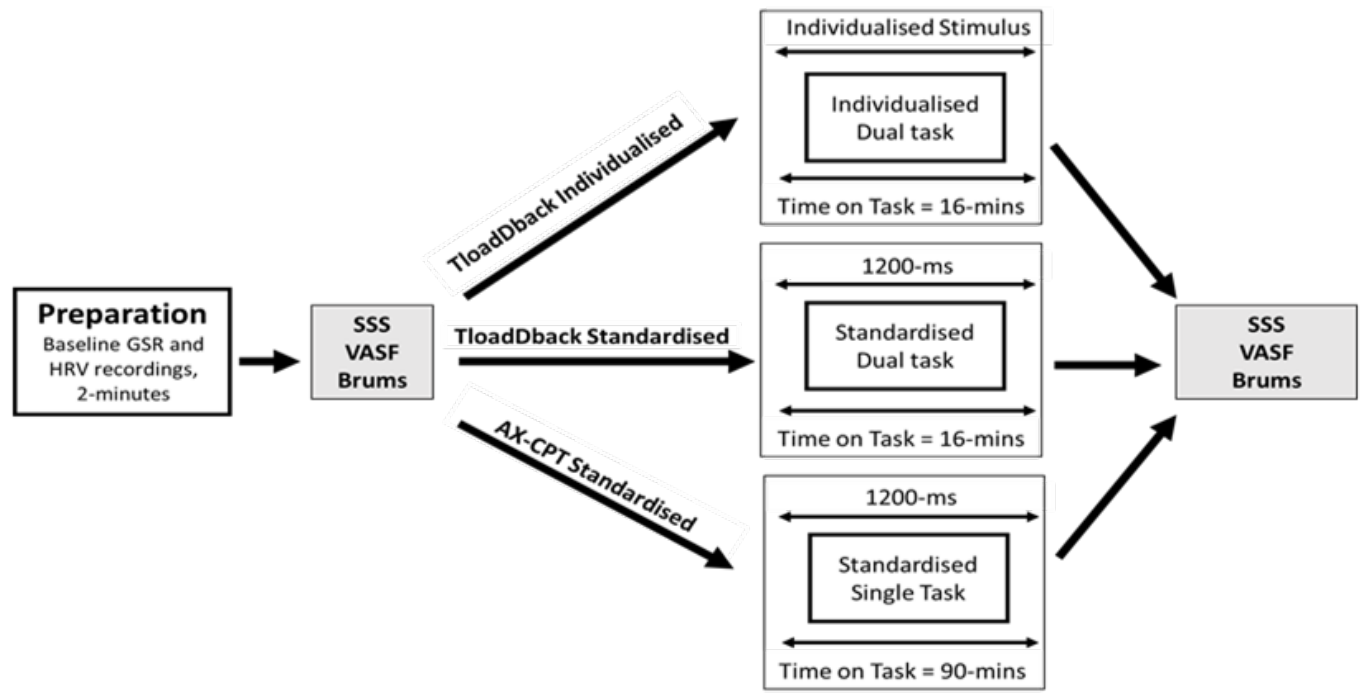


Figure 2.
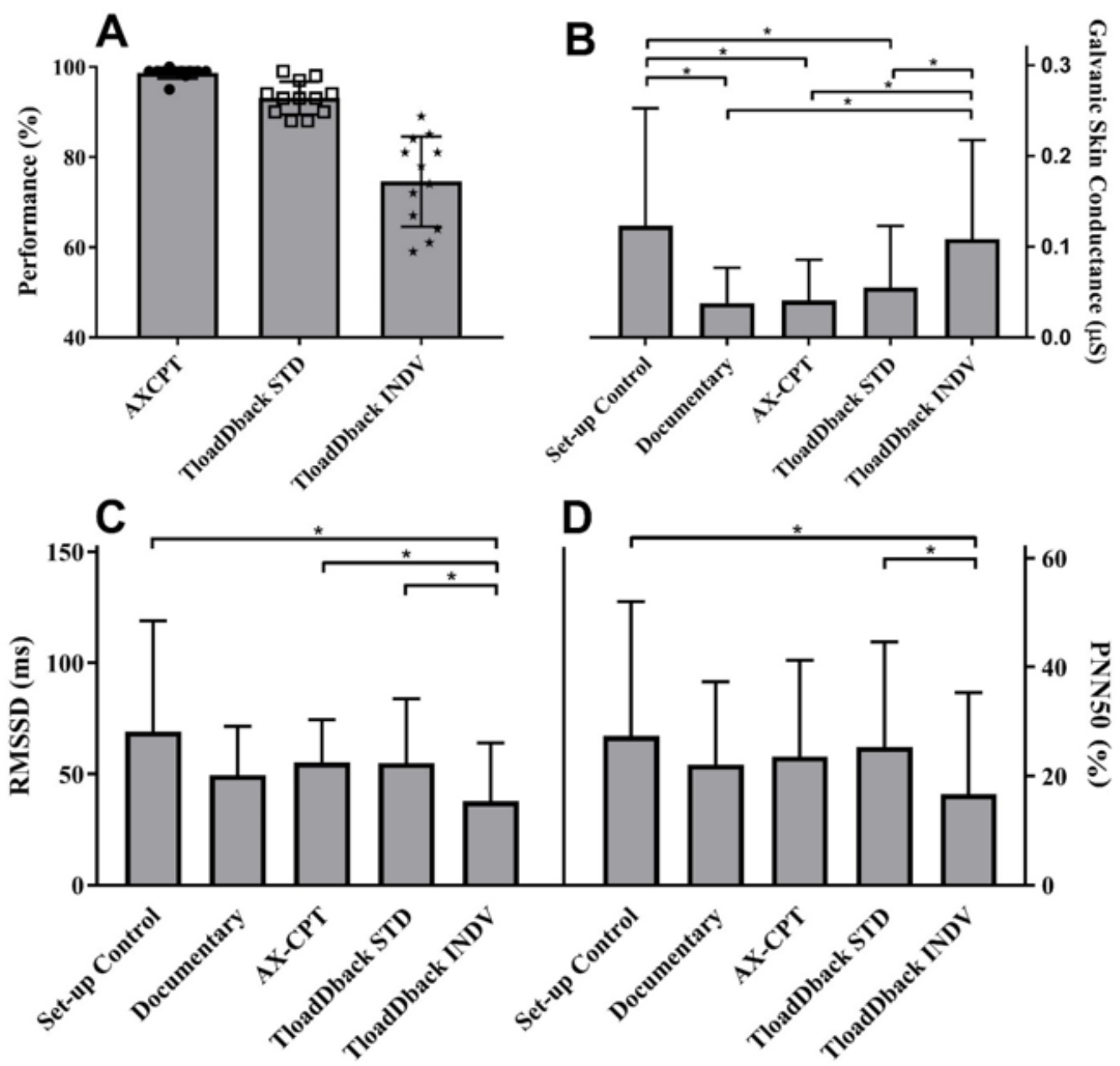


\section{Figure 3.}
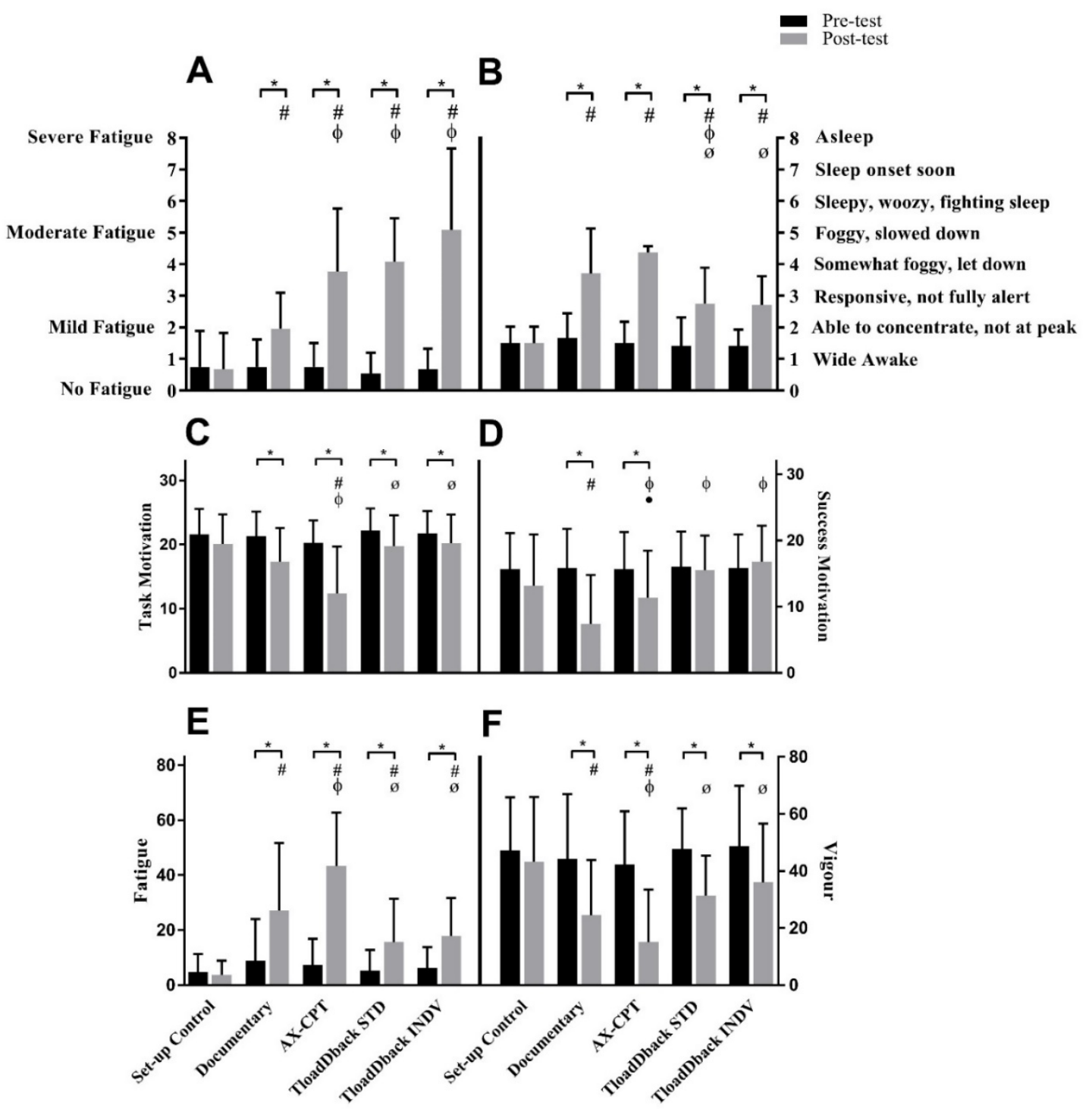

Conditions 